\title{
Space Station GPS Multipath Analysis and Validation
}

\author{
Shian U. Hwu, Y. C. Loh \\ Communications \& Tracking Analysis Group \\ Engineering Analysis \& Simulation Dept. \\ Lockheed Martin Space Operations Company \\ 2400 NASA Rd 1, MC:C80, Houston, TX 77058 \\ Shian.U.Hwu@Imco.com, Y.C.Loh@Imco.com
}

\begin{abstract}
To investigate the multipath effects on the Intemational Space Station (ISS) Global Positioning System (GPS) measurement accuracy, experimental and computational investigations were performed to estimate the carrier phase errors due to multipath. A new modeling approach is used to reduce the required computing time by separating the dynamic structure elements from the static structure elements in the multipath computations. This study confirmed that the multipath is a major error source to the ISS GPS performance and can possibly degrade the attitude determination solution. It is demonstrated that the GPS antenna carrier phase errors due to multipath can be analyzed using the electromagnetic modeling technique such as the Uniform Geometrical Theory of Diffraction (UTD).
\end{abstract}

\section{INTRODUCTION}

The recent progress in the GPS technology results in widely used of the GPS receivers on spacecraft and satellites. The advantages of the GPS over other competing technologies are in the cost, reliability, mass, size, and power consumption, measurement accuracy, and system simplicity.

The International Space Station (ISS) is a very large and complex space vehicle, as shown in Fig. 1. The Global Positioning System (GPS) antennas are surrounded by many large metallic structures such as various solar panels, thermal radiators, Russian Science Power Platform, communications antennas, video cameras and many attached payloads. The solar panels and thermal radiators are rotated dynamically to maintain preferential orientation with respect to the sun. The GPS receiver antennas have to track the GPS Satellites in a wide field of view region and will encounter multipath interference.

The ISS will use the GPS for providing position, velocity, attitude determination, and time reference.
There are concerns about the multipath effects (signal interference due to reflections and diffractions) of the surrounding structure on the GPS performance. Multipath from the surrounding structures may degrade the accuracy of the GPS attitude determination. A series of GPS multipath tests using the ISS GPS hardware were conducted in the NASA/Johnson Space Center (JSC) GPS test facilities. Computational investigations were also performed using the electromagnetic modeling technique-the Uniform Geometrical Theory of Diffraction (UTD). A new modeling approach is used for the ISS GPS multipath modeling and analysis. This approach reduces the required computing time by separating the dynamic structure elements from the static structure elements in the multipath computations.

\section{GPS ERROR SOURCES}

Many error sources affect the GPS carrier phase measurements. There are satellite orbit error, satellite clock drift, ionospheric and tropospheric propagation delays, receiver dynamic tracking error, receiver thermal noise, jamming and interference, antenna phase center movement, and multipath error. For two recciver antennas separated by short baselines, satellite orbit error, satellite clock drift, ionospheric and tropospheric propagation delays errors are common to both antennas. A differential processing (DGPS) can effectively eliminating or greatly reducing these common errors. The receiver dynamic tracking errors, the receiver thermal noise, jamming and interference, antenna phase center movement, and multipath errors can not be removed by the DGPS. The receiver dynamic tracking errors, and the receiver thermal noise can be minimized by the implementation of the receiver design and are predictable for a giving platform dynamics.

Signal Jamming and Interference 
There is considerable concern about the effects of intentional and unintentional signal jamming and interference on critical GPS navigation applications. There is evidence, as well as tests results, to indicate that a problem exists in this regard. The degree of interference to the GPS receiver is a function of the frequency of the interference signal (it must be at or close to the GPS carrier frequencies or its harmonics), the distance from the jamming transmitter, and its power. There are reports that indicate the occasional loss-of-lock on the GPS signals due to the suspicious TV and microwave transmission towers, and various types of radar.

Since the GPS signal formats are well known, GPS receivers are vulnerable to intentional jammers. The only way to protect GPS receivers from intentional jammers is to implement an adaptive nulling array antenna to prevent the interference signals from entering the GPS receiver. This implementation is necessary for the military GPS receivers and may not be necessary for the commercial GPS equipment. For the unintentional interference, many incidents have been reported. Potential interference sources are high power radar, radionavigation transmitters, amateur radios, TV transmitters and co-located radiowave transmitters. Most of the unintentional interferers can be prevented by appropriate filtering implementations.

\section{Antenna Phase Center Stability}

All GPS measurements relate to the distance from the electrical center of the satellite's transmitter to the electrical center of the receiving antenna. Ideally the physical center should coincide with the electrical center, however there is offset for any antenna designs. Because the electrical center varies with the direction and strength of the incoming signal, a variation in the satellite-receiver geometry will cause the position of the electrical center to vary. Phase center stability is an important characteristic for GPS antenna design. Test data have shown that smaller phase center shift for a compact size antenna than a larger volume antennas.

\section{Multipath Errors}

The subject of multipath and mitigation technique for reducing errors has been extensively investigated by many researches. Multipath is receiver-satellite geometry dependent. There is no simple mathematical model to determine or predict the effect of multipath on a position solution. Multipath will bias the baseline results as a function of the multipath signal amplitude and phase relative to the direct signal. The multipath errors are environment dependent and difficult to quantify. The multipath errors are different for each GPS antenna installations and can not be eliminated by differencing techniques. Thus, multipath becomes the dominant error source for high precision GPS applications. Attitude determination using GPS is an important applications for modern spacecraft. The limiting factor is the multipath which accounts for most of the total error budget.

\section{Multipath Mitigation}

Practical mitigation techniques for reducing the multipath errors arc:

- Selecting GPS antenna site to minimize significant reflective ray impinging into the antenna.

- Designing the GPS antenna to maximize RHCP/LHCP ratio which increases multipath rejection capability.

- Using appropriate ground plane or choke-ring assembly to minimize the multipath signals impinging from back and low elevation of the GPS antenna.

- Applying absorber material or paint/coating on the structures where reflections occur.

- Shaping antenna pattern to have high gain in directions where direct signals arrived and to have low gain in multipath signals arriving directions.

- Multipath compensation tables based on electromagnetic modeling technique which may require considerable memory and a match fit to an estimated profile.

- Design a receiver capable of filter out the effect of multipath signal.

- Software mask out the low elevation GPS satellites (signals are more likely to cause multipath).

\section{GPS ANTENNA DESIGN CONSIDERATIONS}

The ISS GPS system will operated in a multipath environment, the GPS antenna plays an important role in minimizing multipath effects and reducing the system errors. For the attitude determination applications, at least 4 GPS satellites in-view for all 4 GPS antennas are required. The omnidirectional antennas are required for obtaining broad coverage and good Geometric Dilution of Precision (GDOP) for the ISS GPS receiver. Unlike ground communications in which users orientation is usually known (normal to the ground), the spacecraft orientation or flight attitude 
can be very different. A linear polarized antenna gives worse polarization mismatch loss. The multipath (reflections and diffractions) causes polarization shift which further complicates the polarization mismatch issues. To avoid potentially severe polarization loss due to misalignment of linear antennas, circular polarization is preferred to ensure the GPS performance in the multipath environment. A low profile antenna gives smaller phase center deviation. This can reduce the measurement errors due to the antenna phase center movements. An ideal antenna with excellent multipath rejection capability should have the following characteristics: (1) nearly hemispherical RHCP pattern (2) with sharp pattern cutoff right above the horizon (3) with very small backlobes (4) with stable phase center over the entire coverage area (5) good axial ratio to provide maximum LHCP multipath signal discrimination.

\section{MULTIPATH GROUND TEST}

\section{Experimental Investigations}

A series of GPS multipath ground tests that simulated the ISS GPS multipath environment were performed. Figure 2 shows the test setup. Each GPS receiver had a laptop computer to record the data. Data were collected with no intentional multipath producers and with intentional multipath producers (plate and cylinder) in place. Only selected data are presented here. For Julian Day (JD) 059, a $4 \mathrm{ft}$. by $12 \mathrm{ft}$. aluminum plate (multipath producer) was placed near the GPS antennas. For JD074, a $3 \mathrm{ft}$. diameter, $4 \mathrm{ft}$. tall cylinder was placed vertically near the GPS antennas.

The multipath effects from the multipath producers were measured as differential carrier phase errors which are the differences between the differential carrier phases measured by the GPS receivers with and without the multipath producers in place. The differential carrier phase errors are presented in millimeters (mm) as a function of time, given in GPS time of week in hours. The $190.5 \mathrm{~mm}$ wavelength $\lambda$, at the GPS L1 frequency of $1.575 \mathrm{GHz}$, corresponds to a 360 degree phase error.

\section{Computational Investigations}

The UTD technique was applied to compute the GPS carrier phase errors due to multipath from surrounding plate and cylinder structures. In the field computation, the reflected, and diffracted fields are determined by the field incident on the reflection or diffraction point multiplied by a dyadic reflection or diffraction coefficient, a spreading factor, and a phase term. The resultant field is given by summing all the complex contributing components:

$$
E^{t o t}=E^{i n c}+\sum_{n=1}^{N} E_{n}^{r e f}+\sum_{m=1}^{M} E_{m}^{d i f}
$$

where $E^{\text {tot }}$ : Total field at the observation point, $E^{\text {inc }}$ : Direct incident field from antenna, $E^{\text {ref }}$ : Reflected fields from plates and cylinders, $E^{\text {dif }}$ : Diffracted fields from plates and cylinders. Detailed information on the theory of this method can be found in [1].

\section{Results and Discussions}

Figures 3 and 4 show the carrier phase errors due to the multipath from a plate near the ISS GPS antennas for satellites 16 and 24 on JD059. The carrier phase errors due to the multipath from a cylinder near the ISS GPS antennas for satellites 1 on JD074 are shown in Fig. 5. The UTD computed GPS carrier phase errors agree well with the experimental test results. Obtained results indicate that structural blockages cause more than $10 \mathrm{~mm}$ or 18.9 degree phase errors on the ISS GPS receiver. Reflections from the structures cause more than $5 \mathrm{~mm}$ or 9.45 degree phase errors.

Both test and computed results indicate that the multipath producers, located near the Space Station GPS antennas, can produce carrier phase crrors of greater than $10 \mathrm{~mm}$ from blockages and $5 \mathrm{~mm}$ from reflections. This study confirmed that the multipath is a major error source to the ISS GPS performance and can possibly degrade the attitude determination solution. It is demonstrated that the GPS antenna carrier phase errors due to multipath from a plate and cylinder can be analyzed using the UTD technique.

\section{ISS MULTIPATH MODELING}

The UTD has been validated with the ground test and is an effective modeling tool for GPS multipath analysis. Since the UTD technique is computationally intensive, the rigorous multipath computations for a complex vehicle like the Space Station poses a challenging task. More than 50 basic structural elements, composed of plates and cylinders, are needed for an Assembly Complete Space Station model. A volumetric pattern at a 2 degree grid density over the GPS field of view will require considerable computing time. In addition, the solar panels and thermal radiators are not stationary. The solar pancls track and thermal radiators antitrack the sun's rays. 
The Space Station configuration thus dynamically changes in the time domain. The conventional UTD computer modeling approach requires recalculating the multipath for all Space Station structural elements at every time step, whenever the solar panels or thermal radiators change their orientations. This approach results in excessive computing time.

A new approach is proposed to investigate the effects of dynamic solar panel and thermal radiator movements on GPS carrier phase measurement. This new approach significantly reduces the required computing time, at every time step, by separating the dynamic structure elements, such as solar panels and thermal radiators, from the static structure elements, such as modules, the Russian power tower, the centrifuge, and the trusses in the rigorous multipath computations using UTD. The total multipath, at every time step, from the Space Station structures is then the appropriate combination of multipath from both the dynamic structures and the static structures.

\section{Results and Discussions}

The accuracy of the results from the new technique is compared with that from using the conventional UTD modeling technique. Figure 6 shows that the computed signal strength (or antenna gain) along a selected satellite path is very similar for both the conventional and new UTD modeling approaches. Almost identical phase shift along a selected satellite path is also observed in Fig. 7. Figure 8 shows that the computed percent coverage for the volumetric antenna gain patterns is very close for both the conventional and new UTD modeling approaches. Figure 9 shows that the computed percent coverage for the volumetric phase shift patterns is only slightly different between the conventional and new UTD modeling approaches. There are significant separations in terms of wavelength between the static structures and the dynamic structures that result in insignificant electromagnetic interactions between them. The difference in the computed antenna gain and phase patterns, using the two different modeling approaches, is small and is mostly in the small phase shift region.

This new approach is accurate and significantly reduces the required computing time by separating the dynamic structure elements from the static structure elements in the multipath computations. Results obtained from this study indicate that this new approach reduces the computing time considerably, by more than $30 \%$, and only very slightly decreases the accuracy of the GPS receiver antenna gain and phasc shift patterns.

\section{REFERENCES}

[1]Marhefka, R. J., and Silvestro, J. W., "Near Zone Basic Scattering Code User's Manual with Space Station Applications," NASA Contractor Report 181944, Dec. 1989.

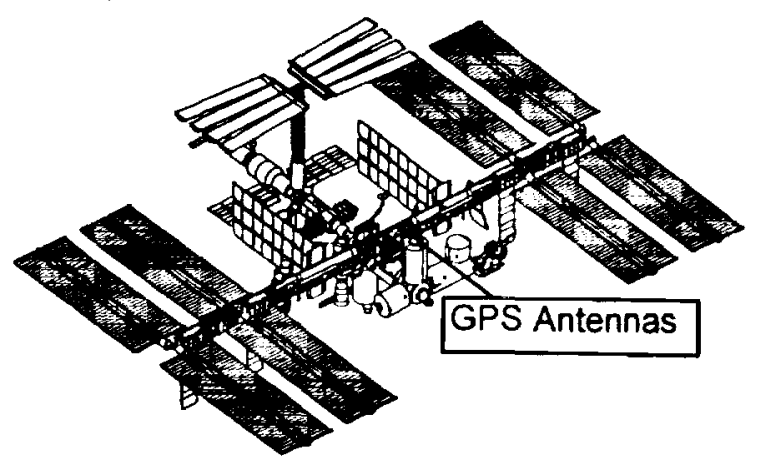

Fig. 1. International Space Station GPS antenna locations.

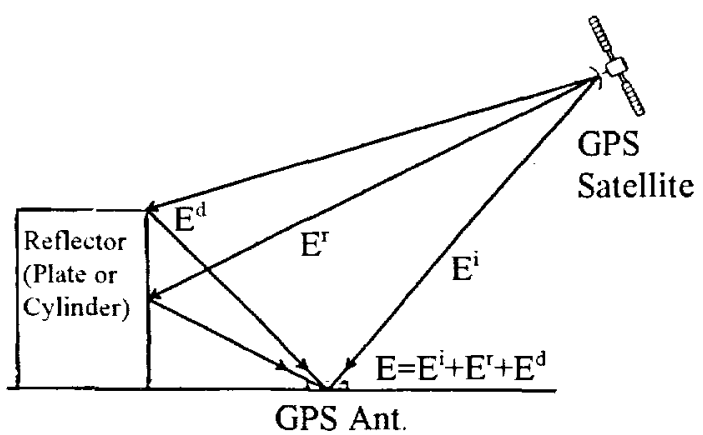

Fig. 2. GPS multipath ground test configuration.

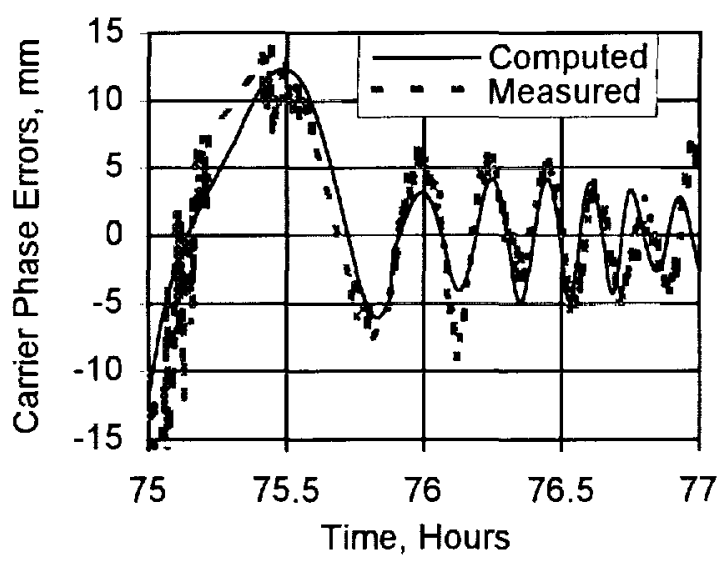

Fig. 3. Carrier phase errors due to multipath from a plate for Satellite 16 on JD059. 


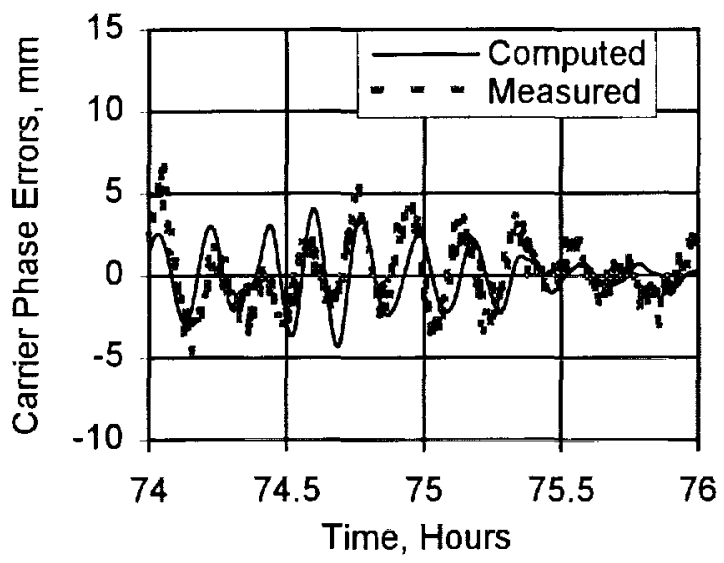

Fig. 4. Carrier phase errors due to multipath from a plate for Satellite 24 on JD059.

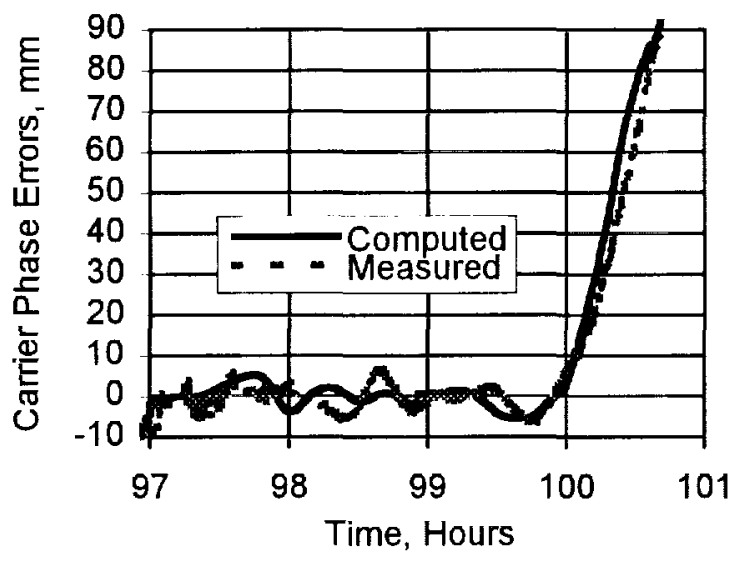

Fig. 5. Carrier phase errors due to multipath from a cylinder for Satellite 1 on JD074.

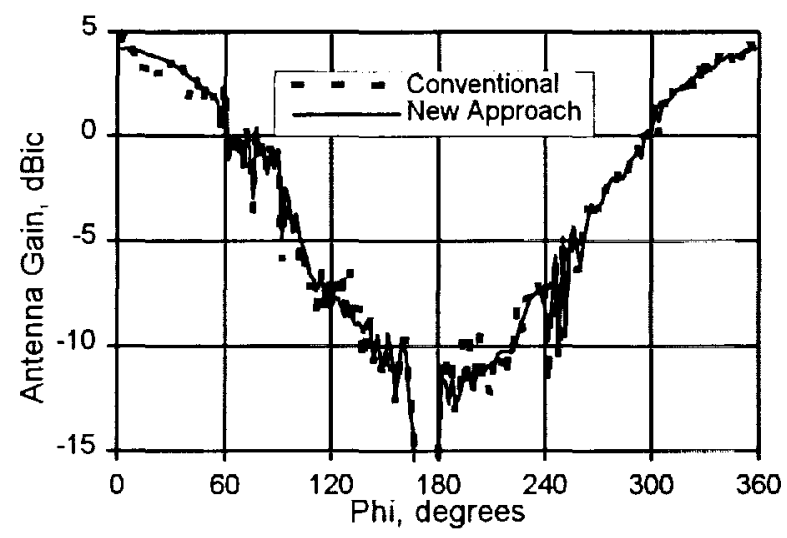

Fig.6. Signal strength comparison along a satellite path.

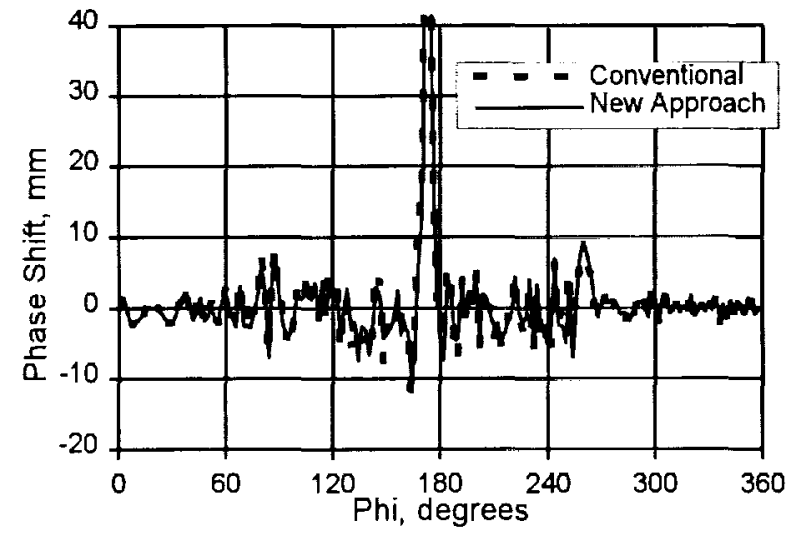

Fig.7. Phase shift comparison along a satellite path.

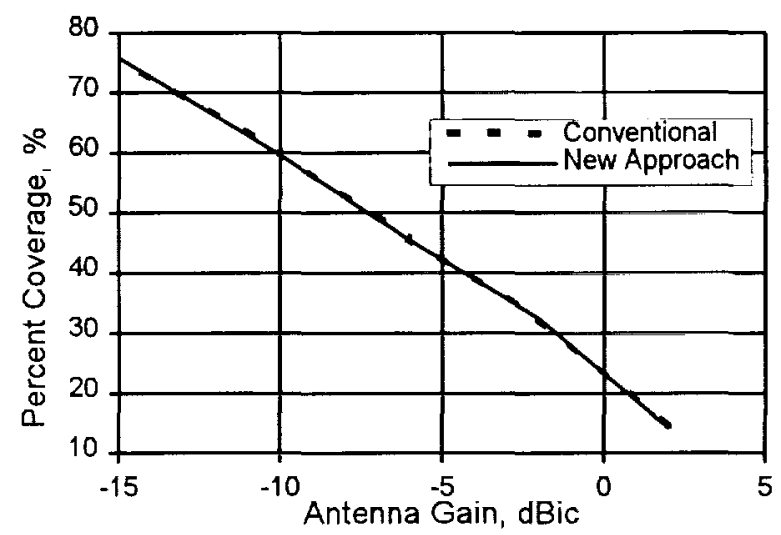

Fig.8. Comparison for volumetric signal strength pattern.

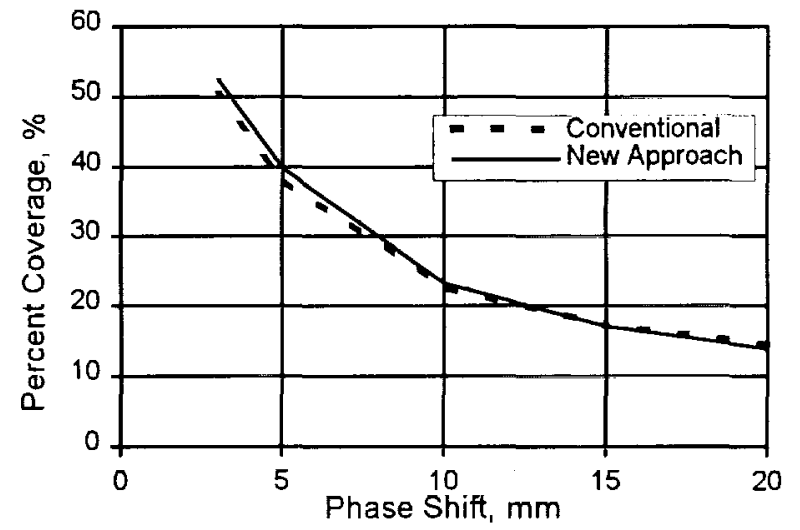

Fig.9. Comparison for volumetric phase shift pattem. 